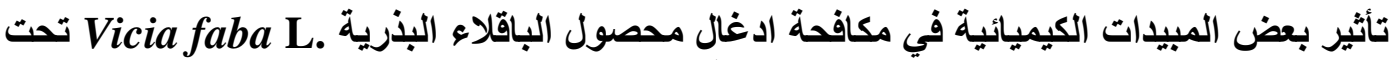 الظروف الايمية في شمال العراق الباق

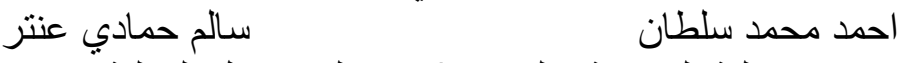 \\ كلية الزر اعة و الغابات / قسم المحاصيل الحقلية
}

\section{الخلاصة}

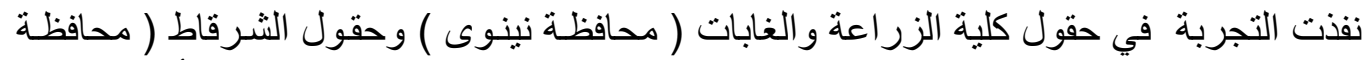

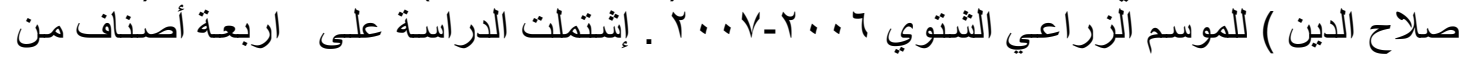

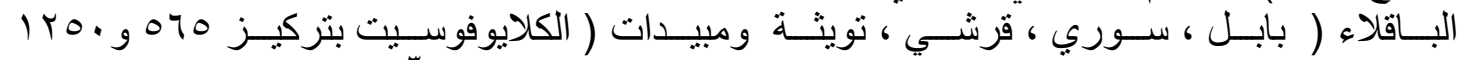

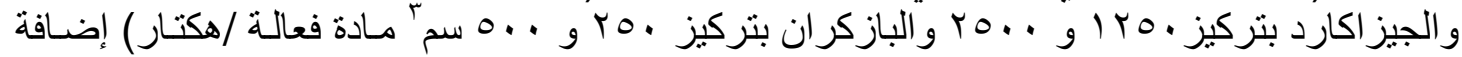

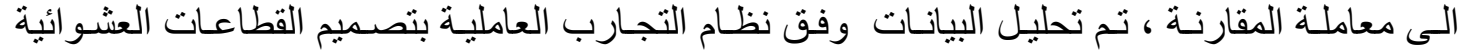

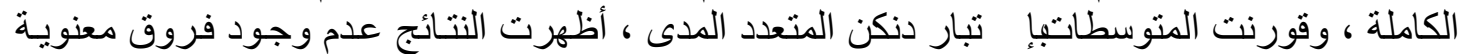

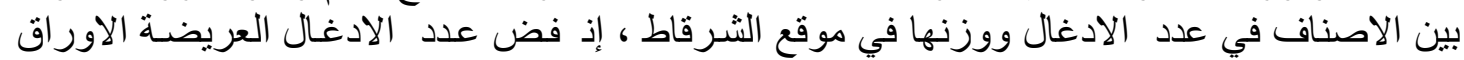

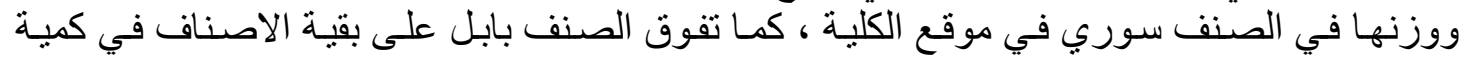

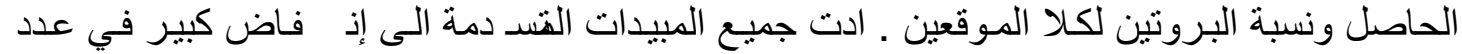

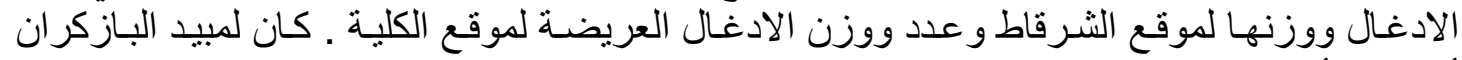

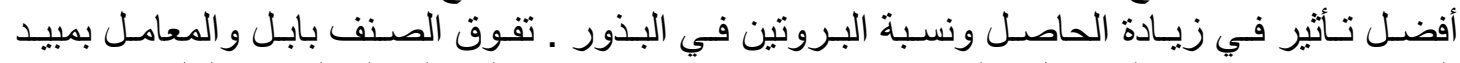

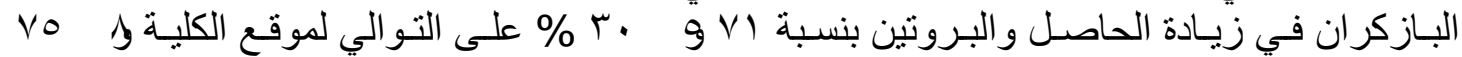

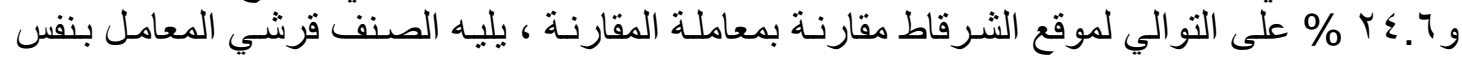

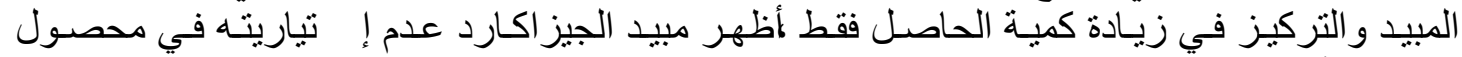

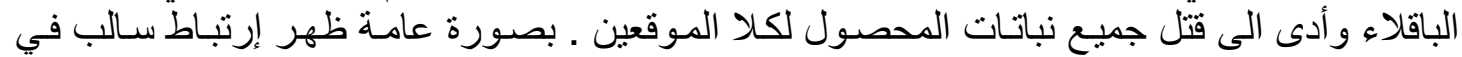

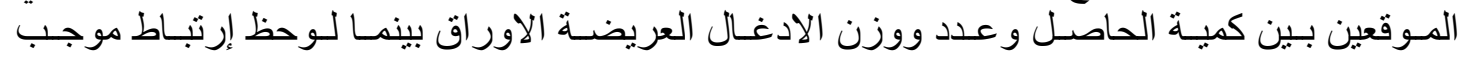

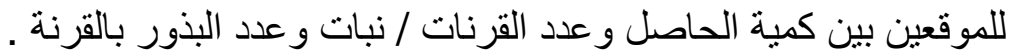

\section{المقدمة}

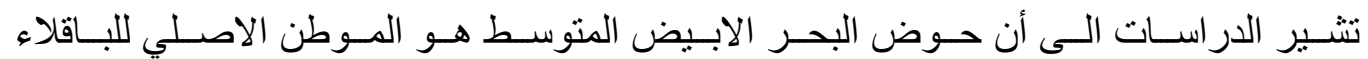

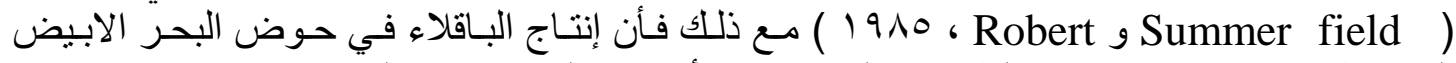

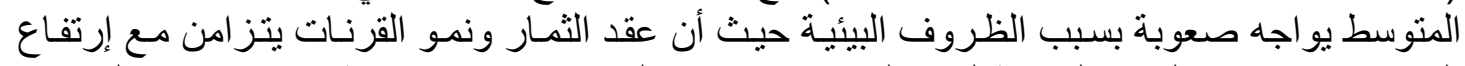

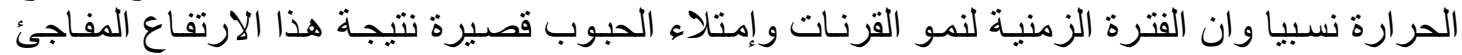

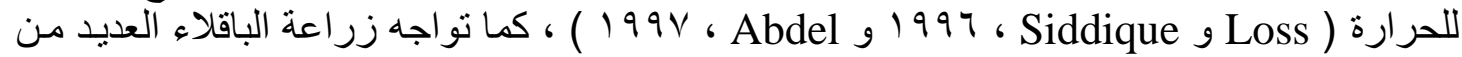

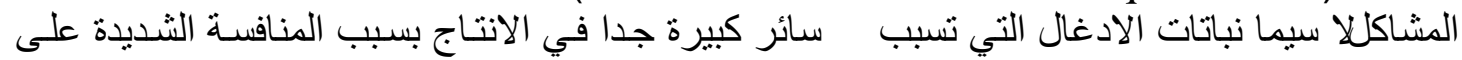

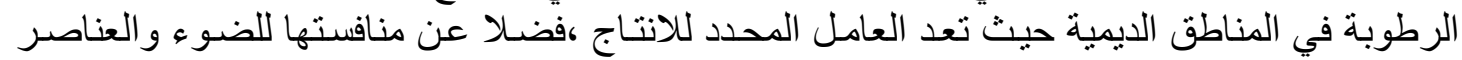

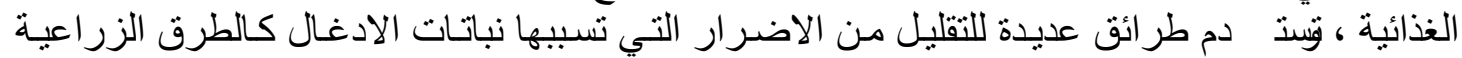

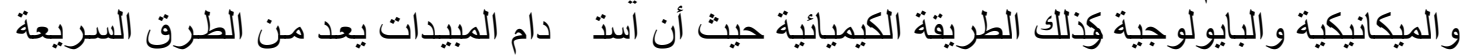

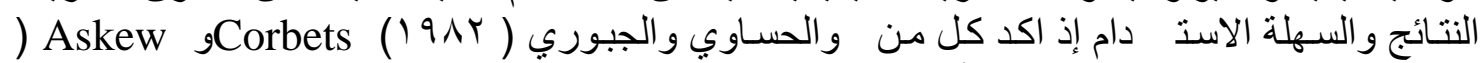

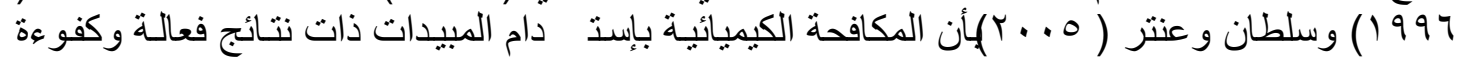

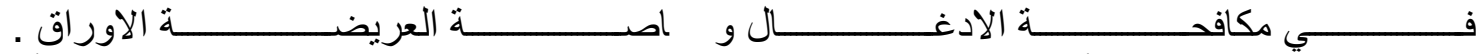

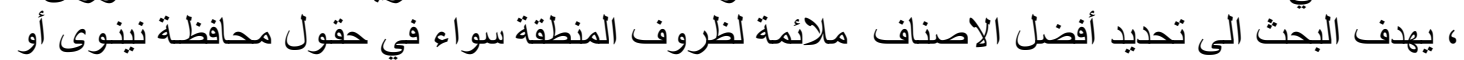

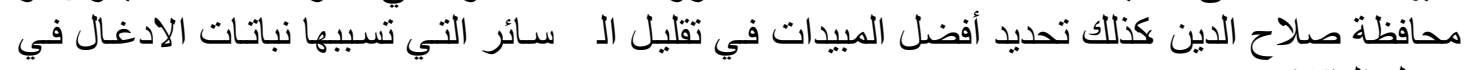
حقول الباقلاء.

\section{مواد البحث وطرائقه}

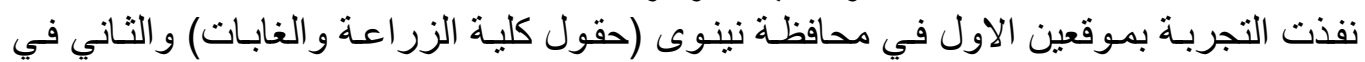

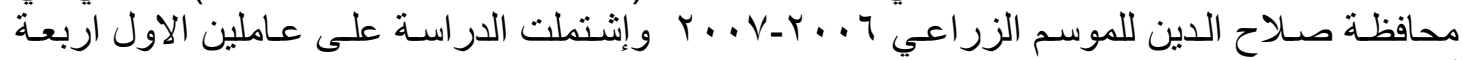

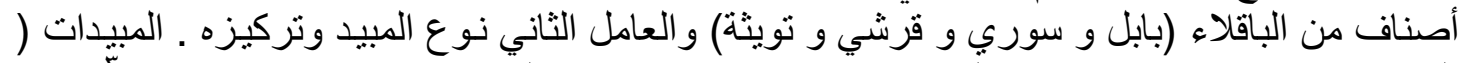

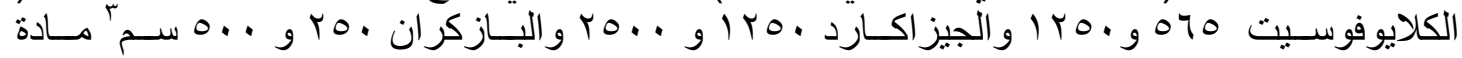

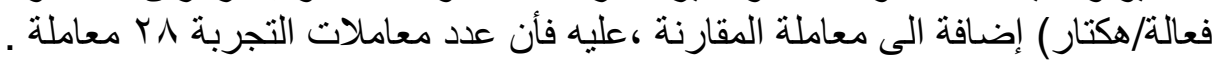




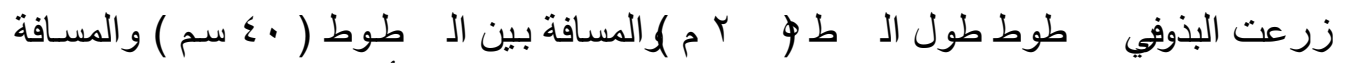

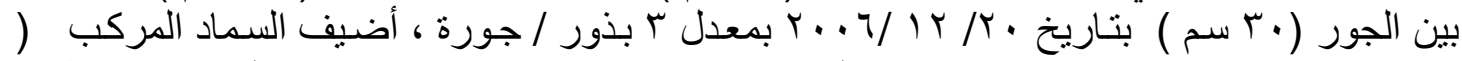

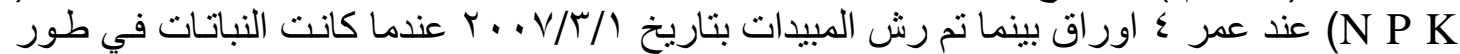

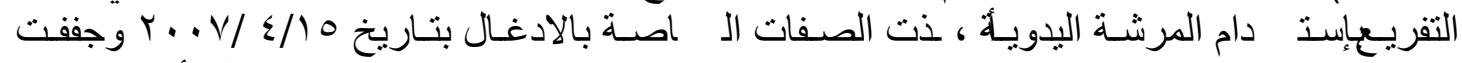

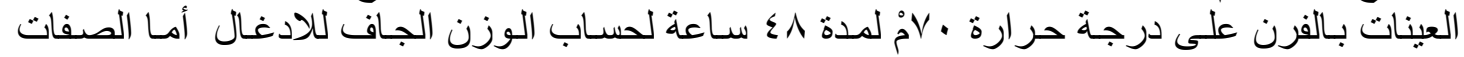

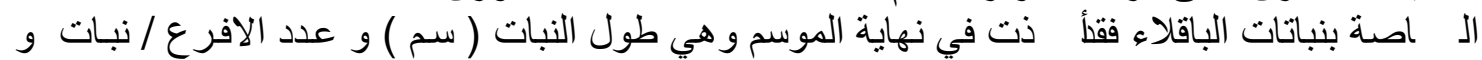

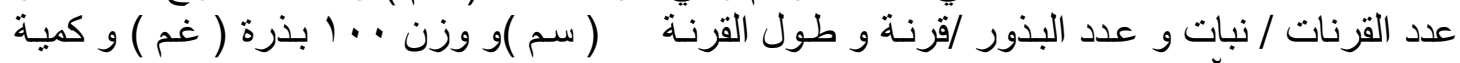

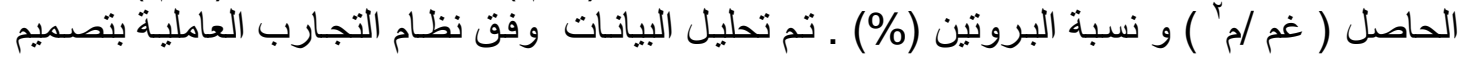

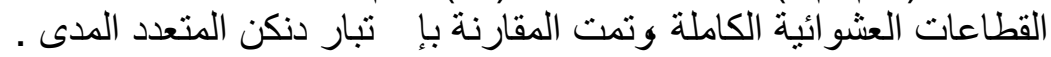

\begin{tabular}{|c|c|c|c|c|c|c|}
\hline \multicolumn{3}{|c|}{ تكريت } & \multicolumn{3}{|c|}{ الموصل } & \multirow{3}{*}{ كانون الثاني } \\
\hline معدل سقوط الأمطار & درجة الحرارة الصغرى & درجة الحرارة العظمى & معدل سقوط الأمطار & درجة الحرارة الصغرى & درجة الحرارة العظمى & \\
\hline Tr & r ro & $\pi$ & $\begin{array}{ll}r q & r\end{array}$ & 1.0 & Ir 09 & \\
\hline 01 & $0 \quad 19$ & $17 \leqslant \varepsilon$ & $7 \varepsilon \quad V$ & 19 & $1 \leqslant \wedge \wedge$ & شباط \\
\hline $17 r$ & $\Lambda$ ro & rY r & TY r & $V \quad .0$ & $191 \leq$ & آذار \\
\hline Tा & 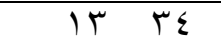 & $\begin{array}{ll}r & 1\end{array}$ & $\begin{array}{rr}r q & r\end{array}$ & $1 . r$ & $\begin{array}{l}Y T \\
Y\end{array}$ & نيسان \\
\hline 1. & $T \varepsilon$ & rV 1 & 19 & $19 \leqslant 1$ & $r \xi \quad \varepsilon$. & مايس \\
\hline
\end{tabular}

\section{النتائج والمناقشة إنة}

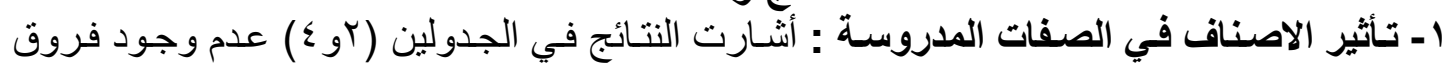

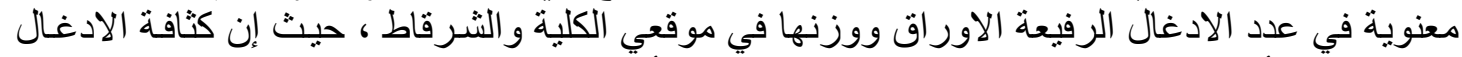

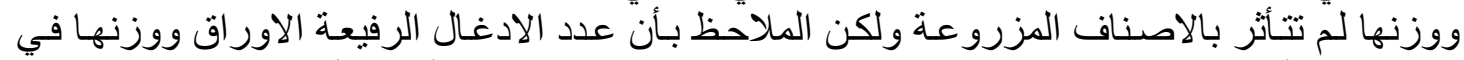

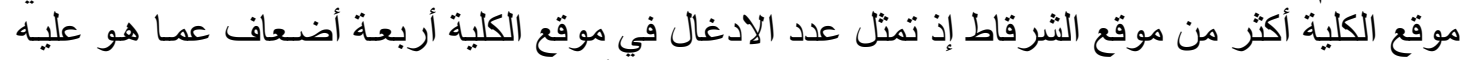

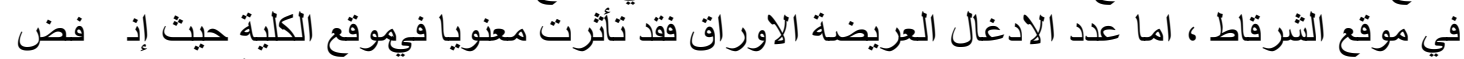

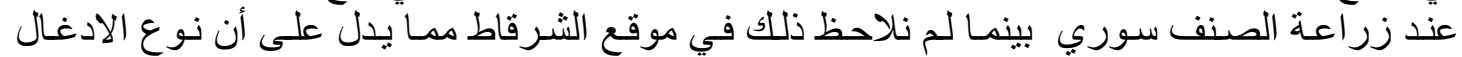

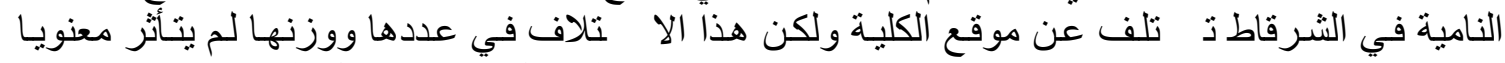

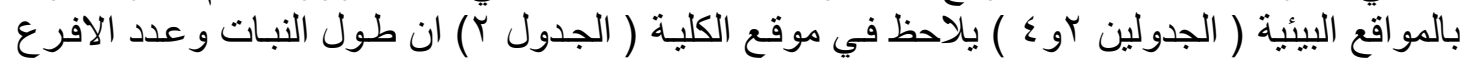

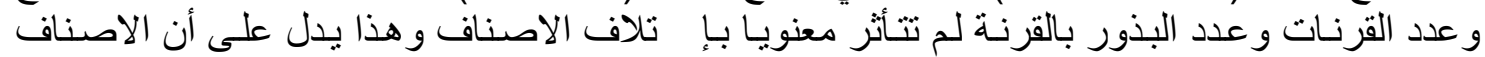

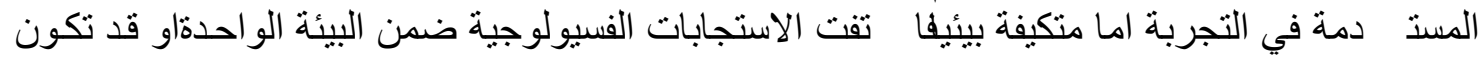

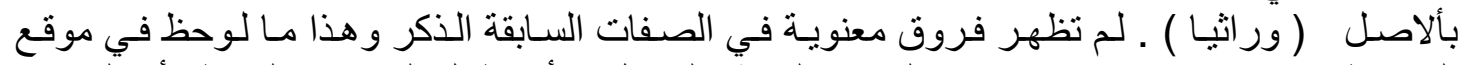

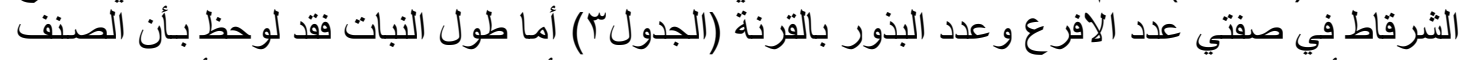

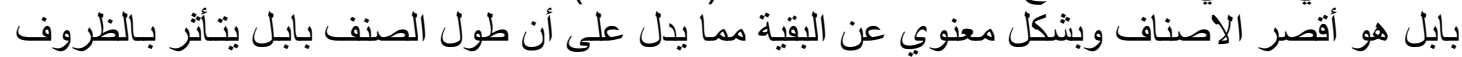

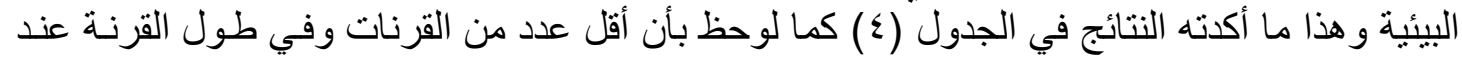

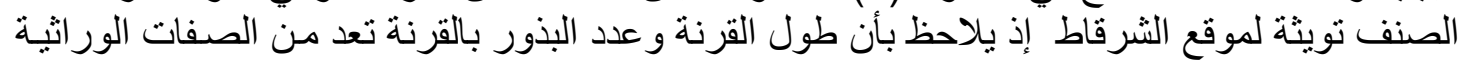

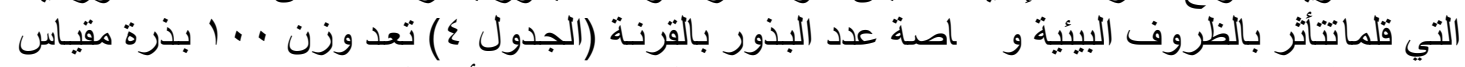

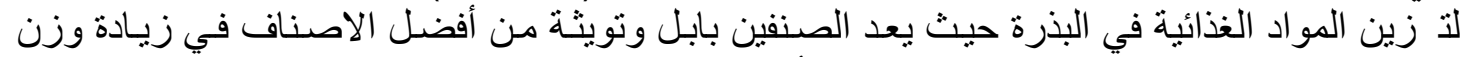

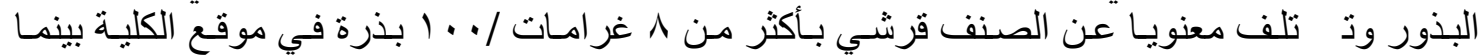

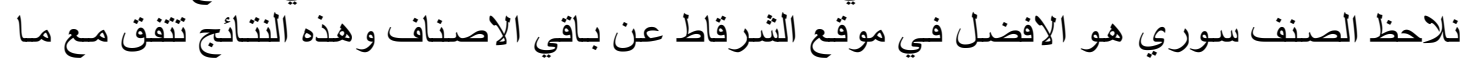

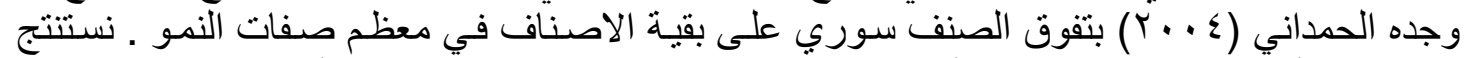

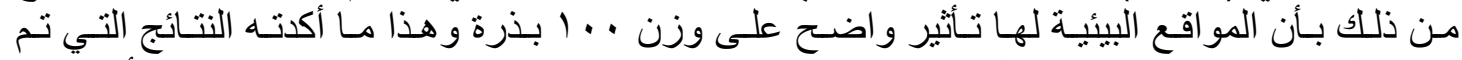

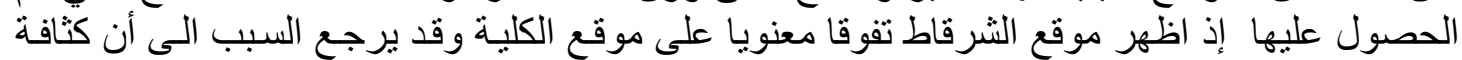

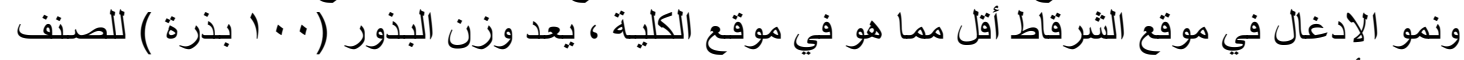

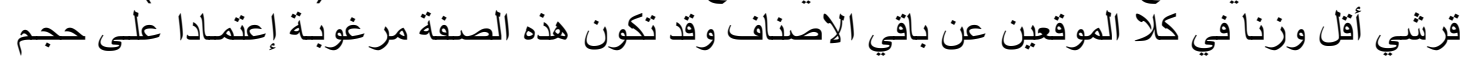




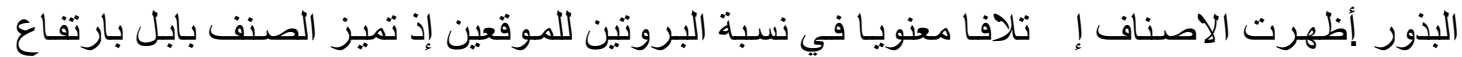

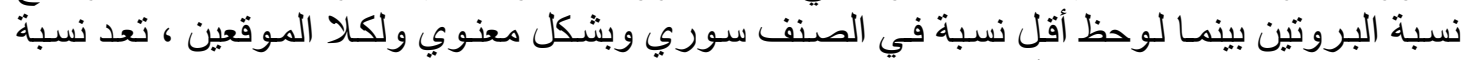

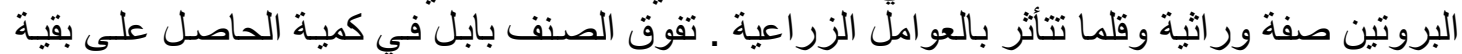

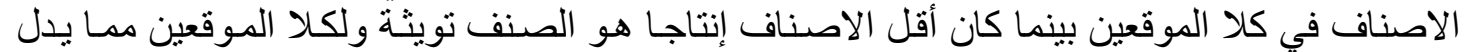

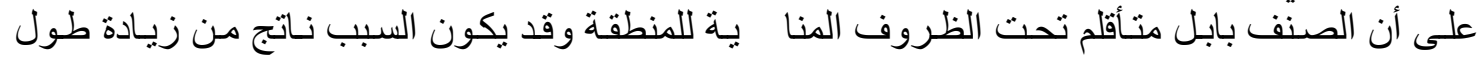

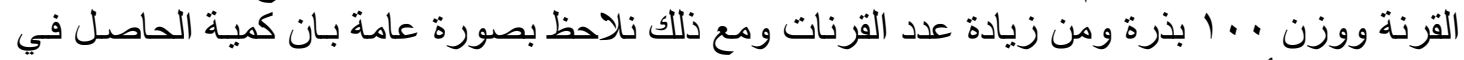
الثرقاط هي أعلى من موقع الكلية.

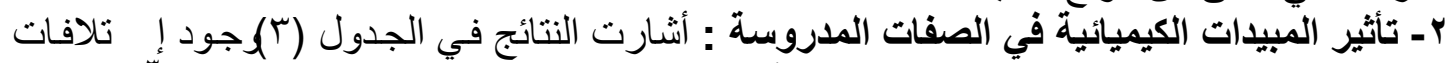

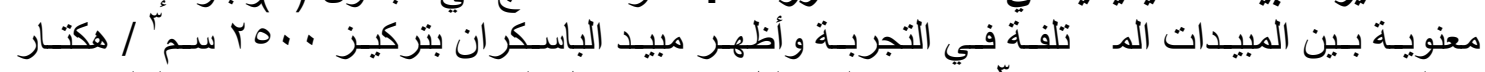

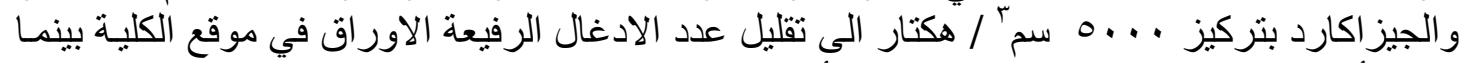

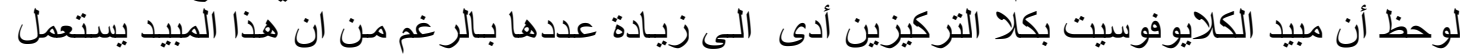

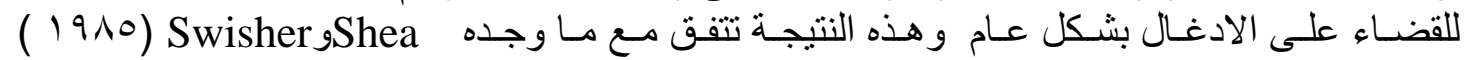
Soutie

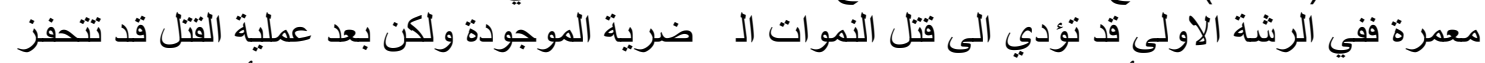

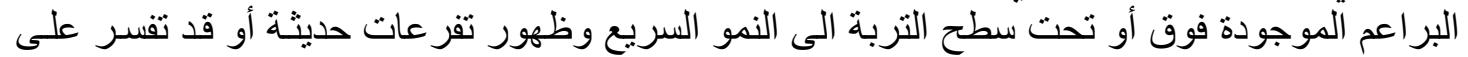

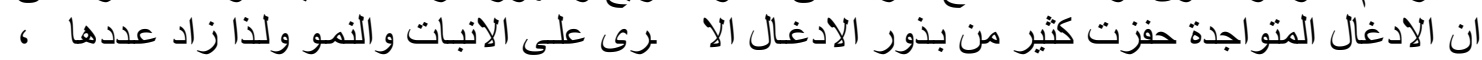

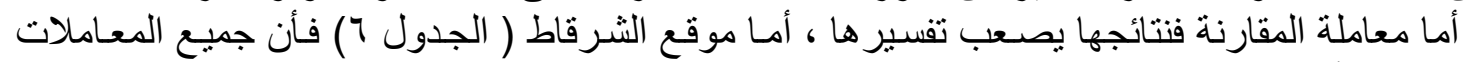

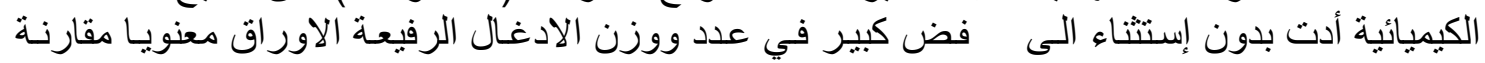

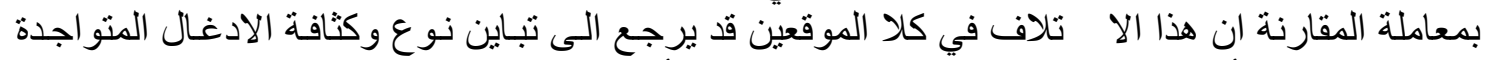

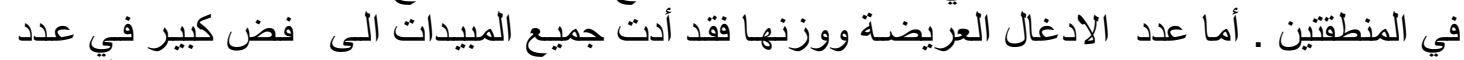

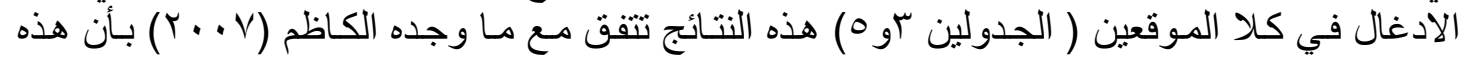

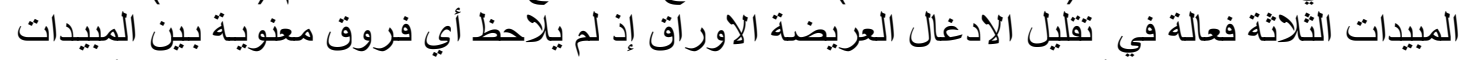

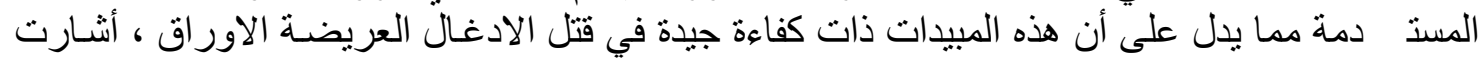

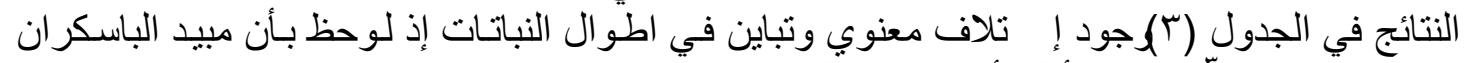

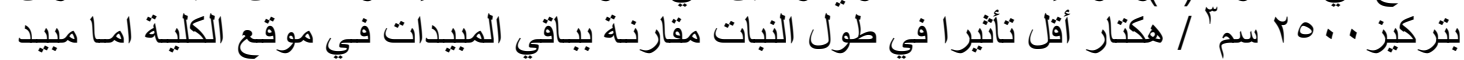

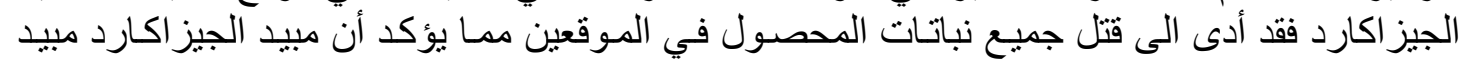

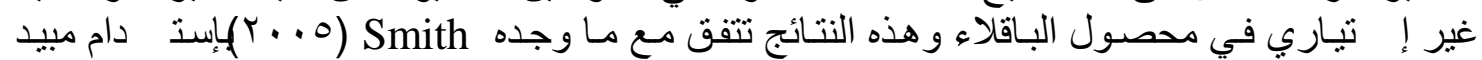

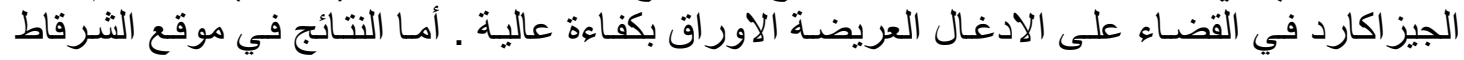

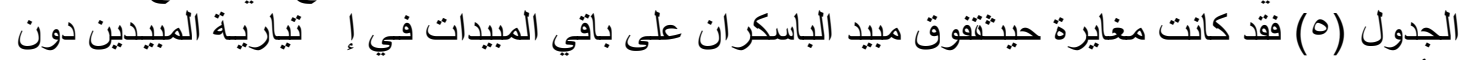

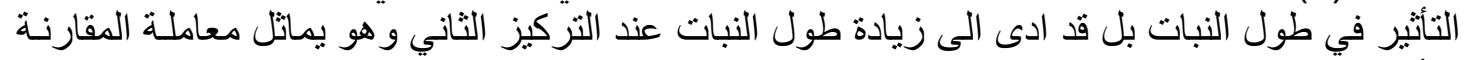

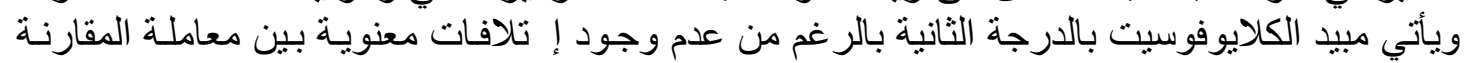

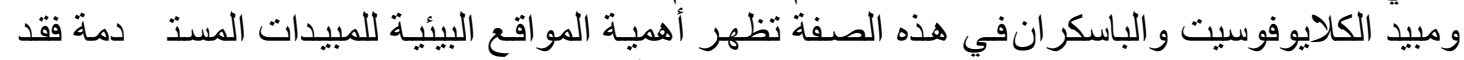

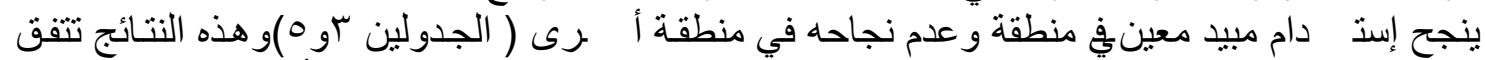

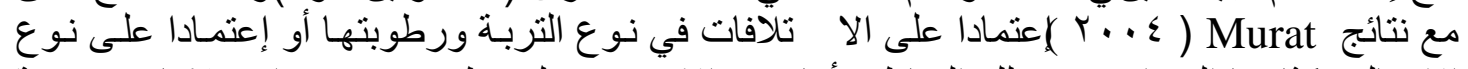

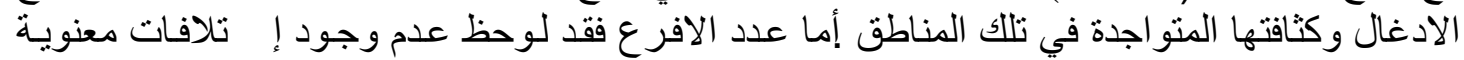

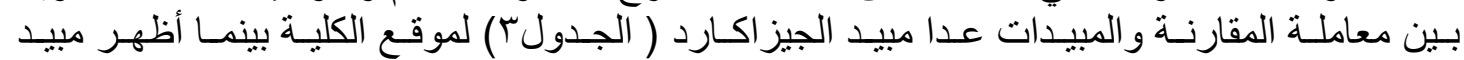

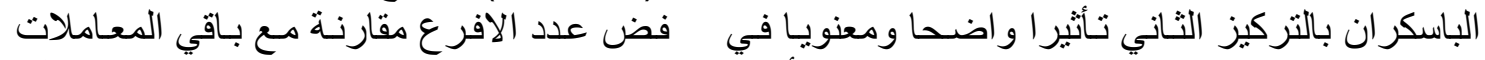

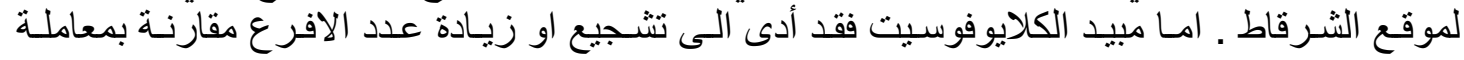
المقارنة .

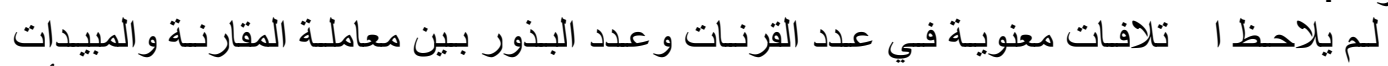

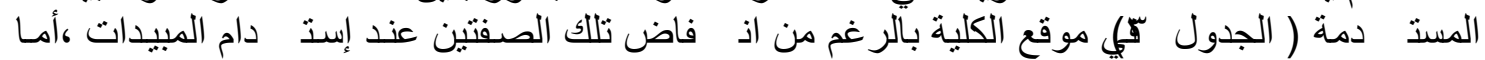

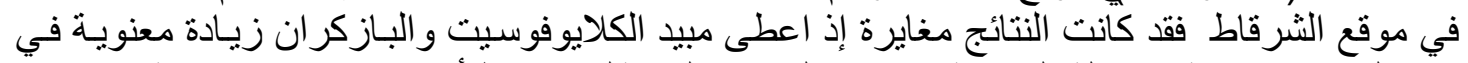

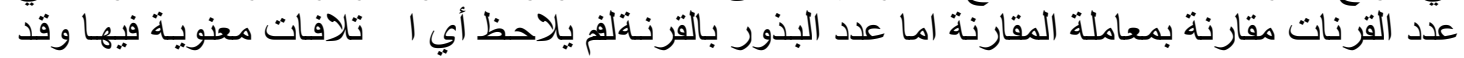

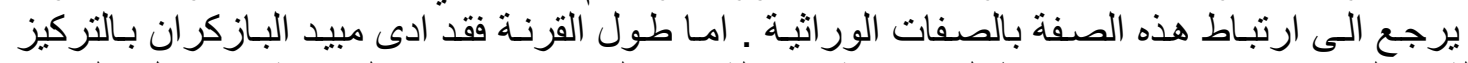

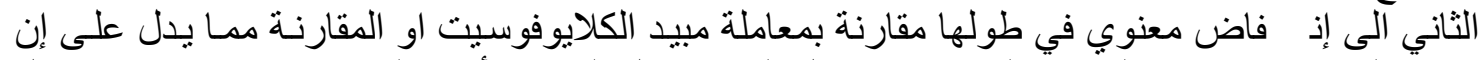

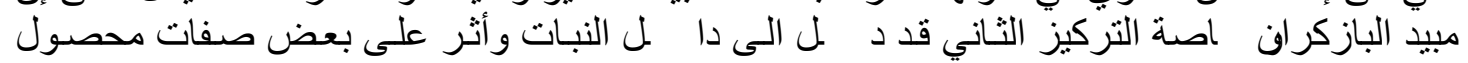




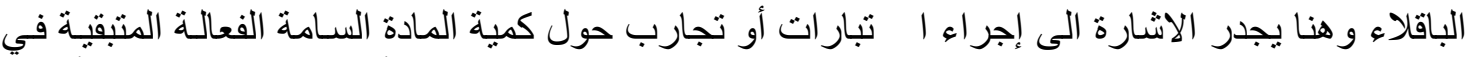

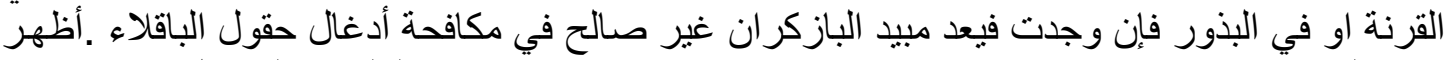

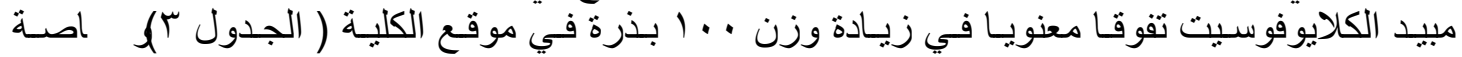

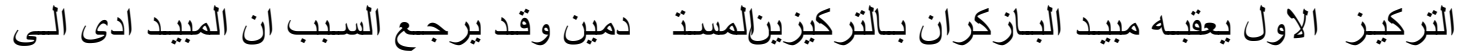

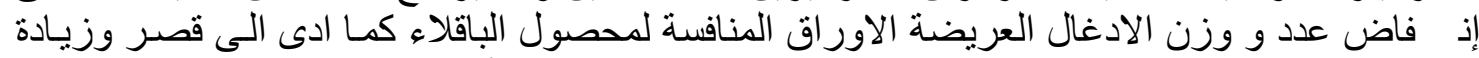

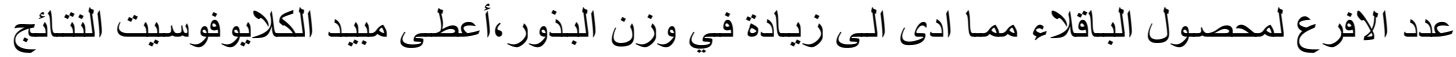

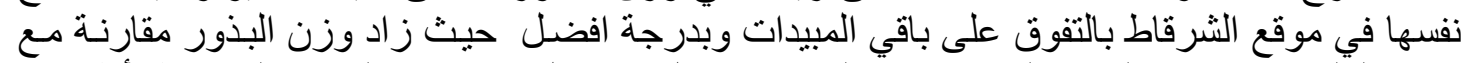

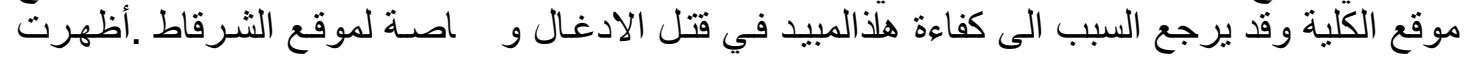

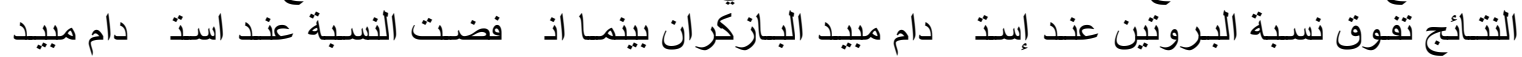

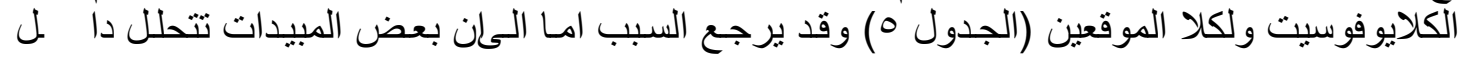

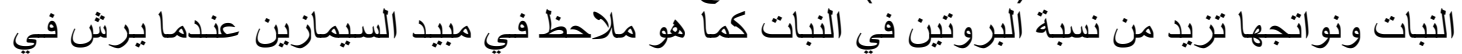

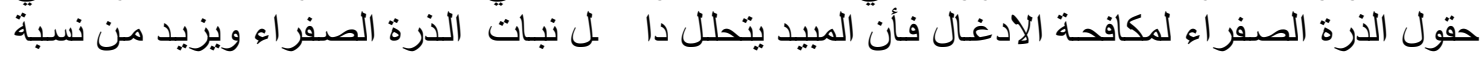

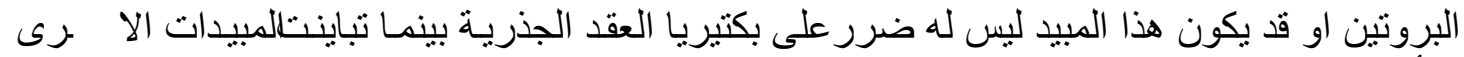

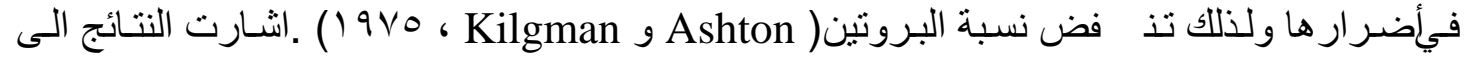

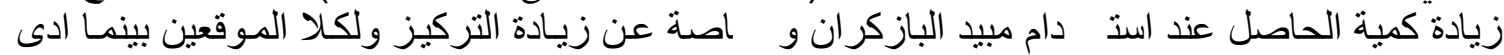

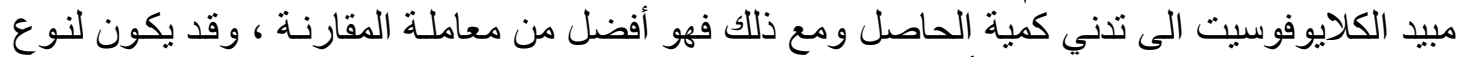

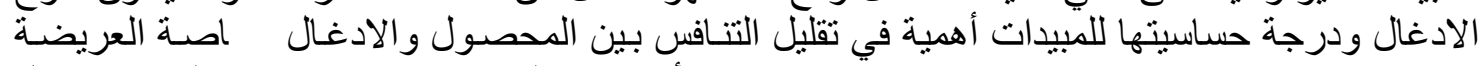

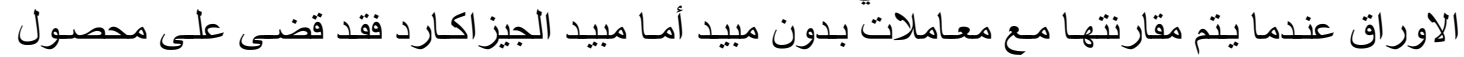

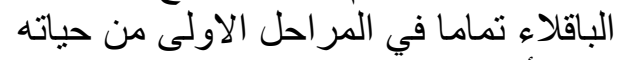

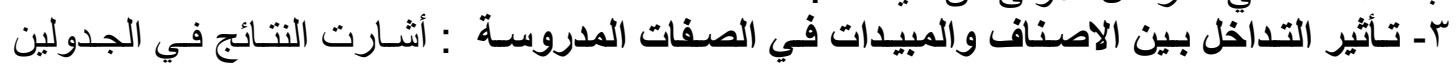

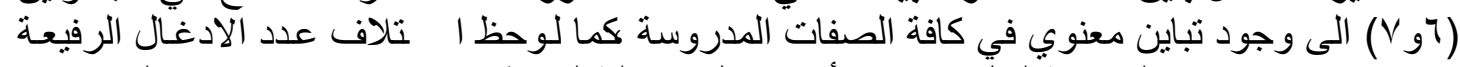

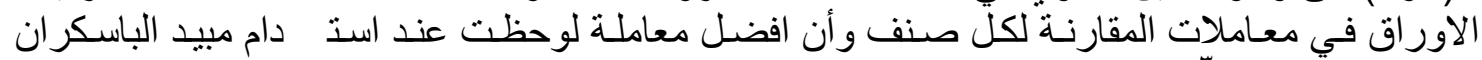

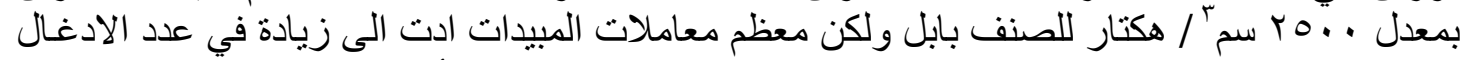

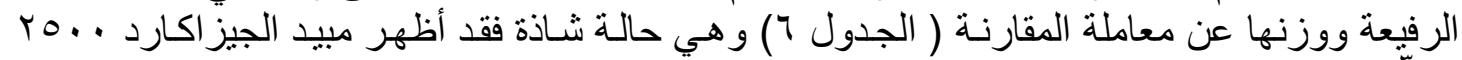

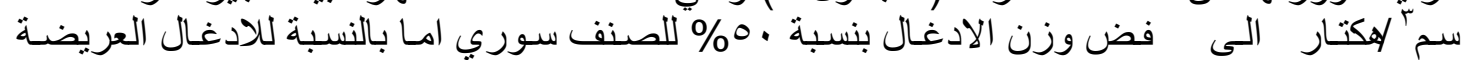

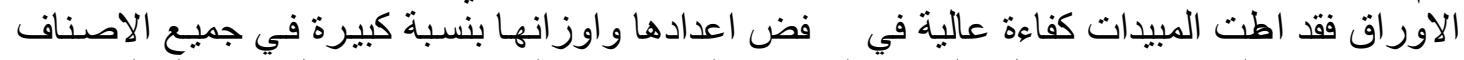

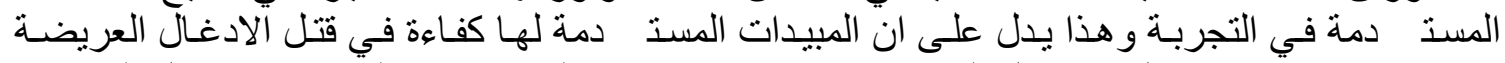

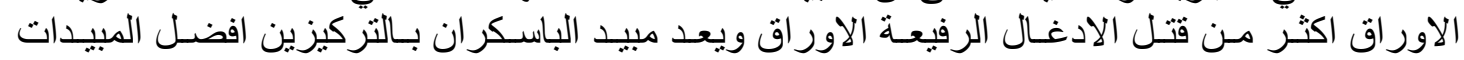

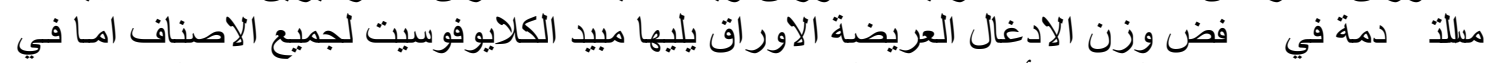

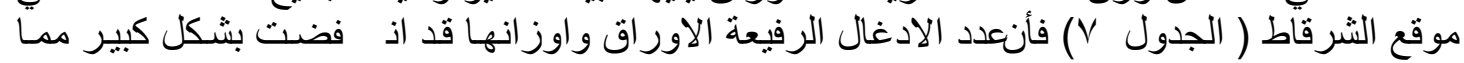

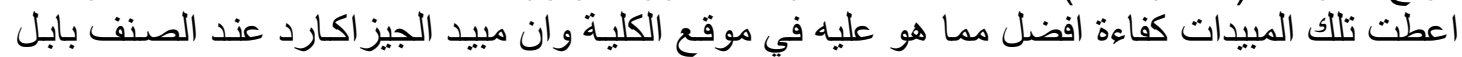

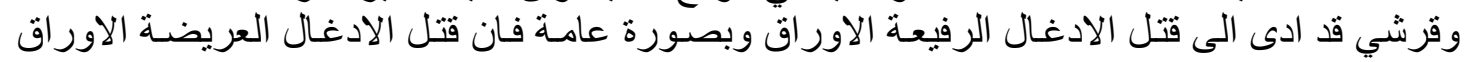

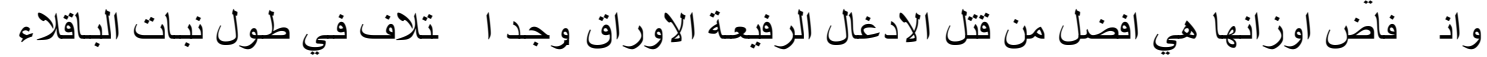

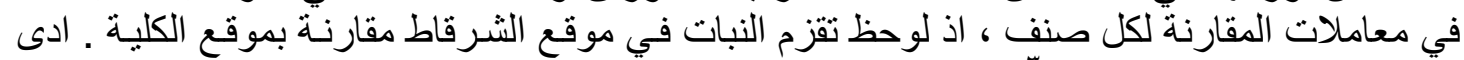

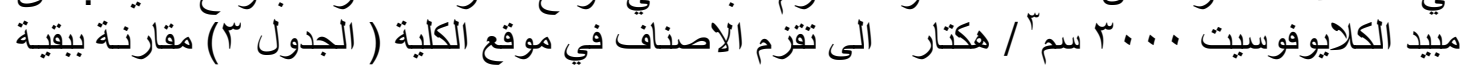

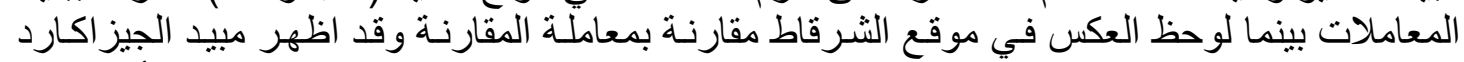

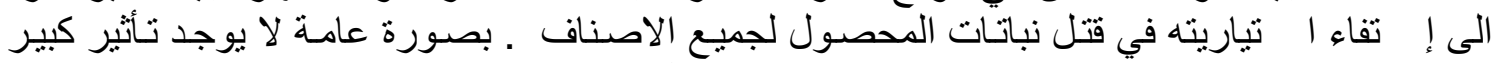

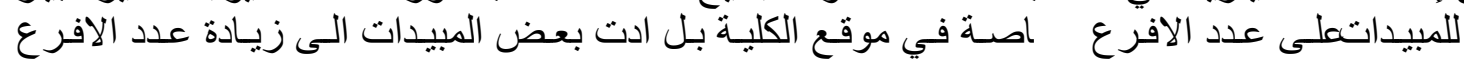

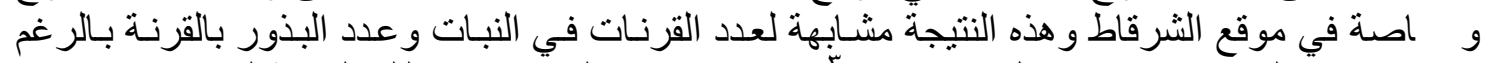

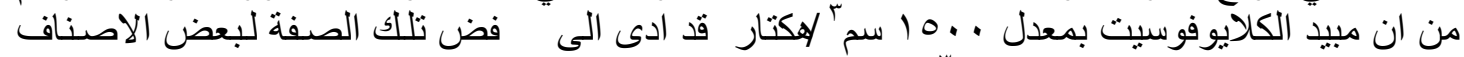

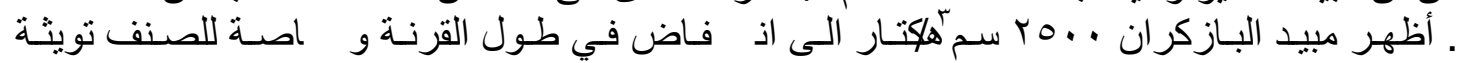

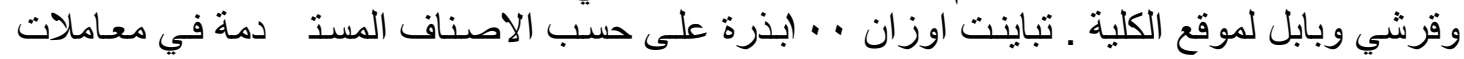

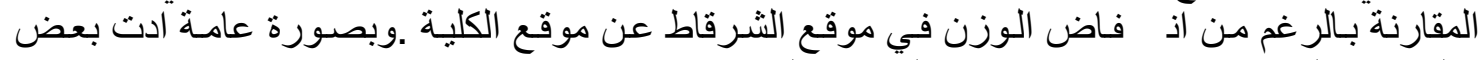

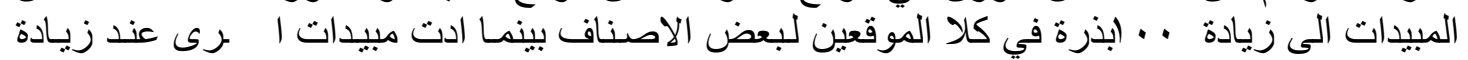

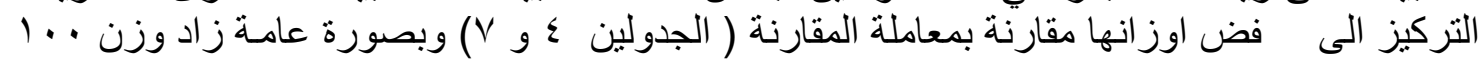

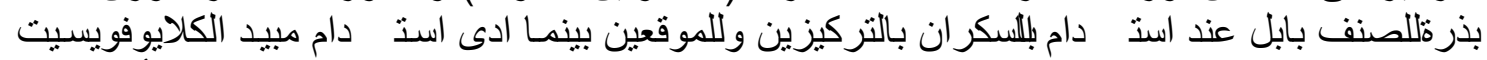

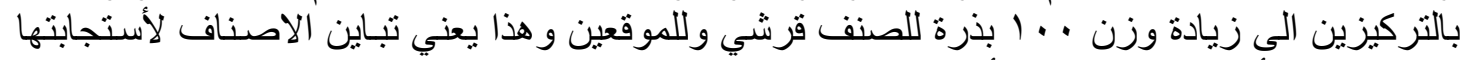

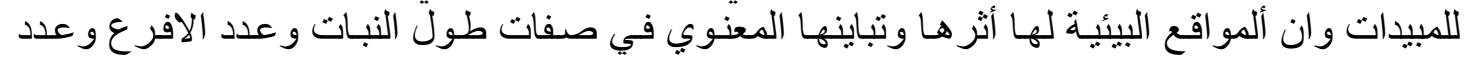




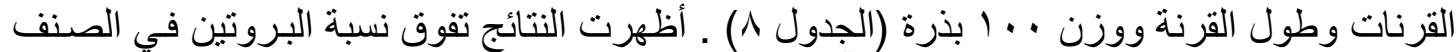

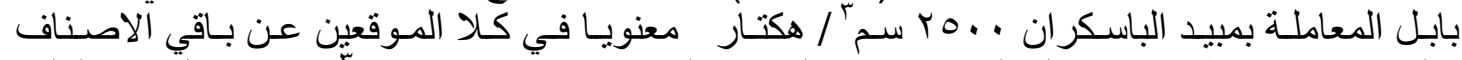

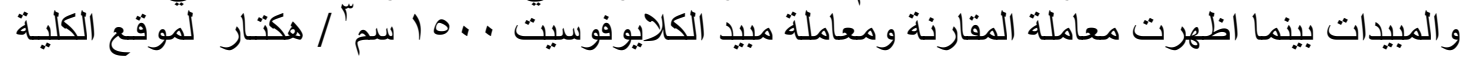

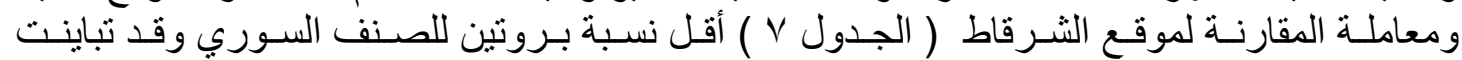

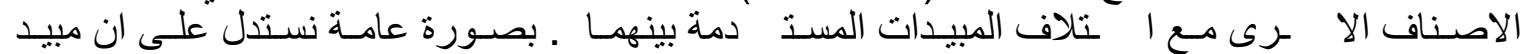

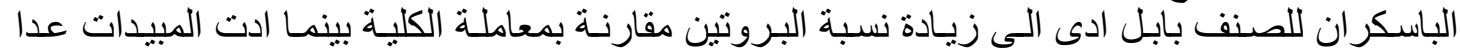

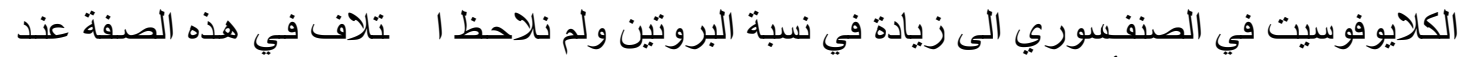

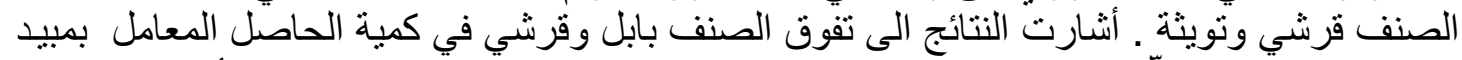

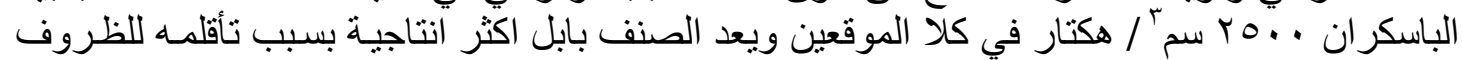

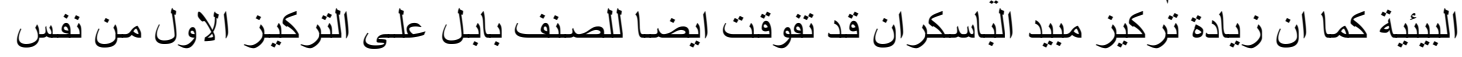

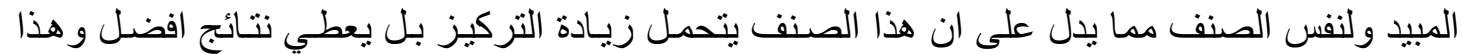

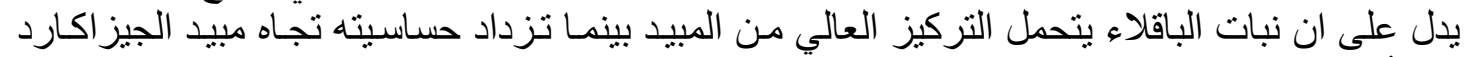

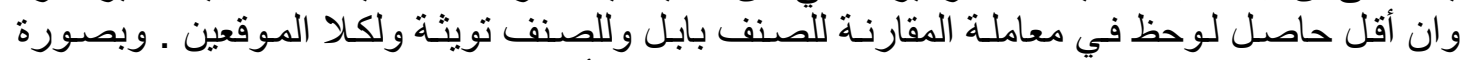

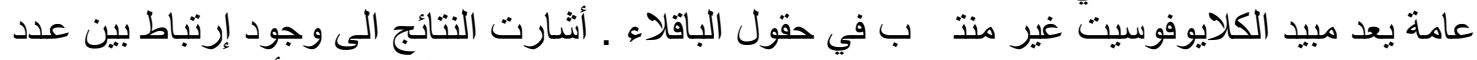

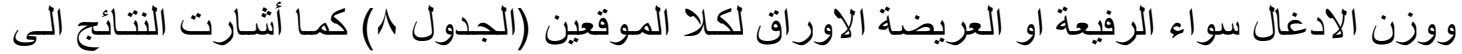

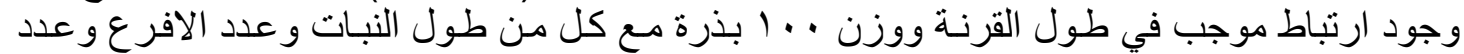

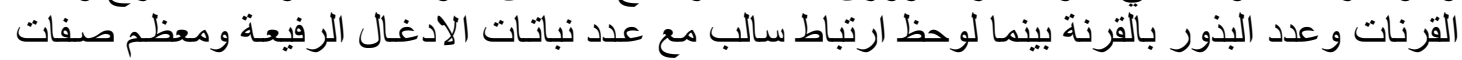
المحصول . المتات

\title{
EFFECT OF SOME HERBICIDES ON WEED CONTROL IN BROADBEAN ( Vicia faba L.)UNDER DRY LAND FARMING IN NORTHEN IRAQ
}

A.M.Sultan

S .H.Anter

Field crops Dept . College of Agric \& Forestry, Mosul Univ., IRAQ

\begin{abstract}
Factorial experiment was carried out at College farm (Mosul prov) and Sharkat (Salah alldeen prov) in 2006-2007 winter growing season to investigate the effect of some herbicides (Glyphosate 565 , 1250 ,Gesagard 1250 ,2500 Basgran $250,500 \mathrm{~cm}^{3}$ a.i /ha and check treatment ) on four varity of broadbean crop ( Babylon, Syrain , Koryshi, Towythai ) . RCBD design with three replications were used with Duncan multiple rang test .Unsignificant results had been showed between varitys in the weed biomass at Sharkat while Syrain varity had reduced the broad leave weed biomass at college farm . On other hand, Babylon varity was superior in the seed yield and protein at two locations. Herbicides used gave excellent reduction in weed biomass at Sharkat location but at college farm ,the reduction had seen on broad leaves weeds only. The interaction between Babylon $\times$ Basagran $500 \mathrm{~cm}^{3} /$ ha gave a good result in increasing seed yield and protein up to 71 and $30.5 \%$, respectively at college farm and 75.8 and $24.6 \%$, respectively at Sharkat location if it compared with check treatment. The second option was with koryshi varity treated with same herbicide and dose in increasing seed yield only . Gesagard had no selectivety for broadbean which all crop plants under land farming. Generally, there is a negative correlation at two locations between seed yield and broad leave weed biomass while positive correlation had been seen between seed yield and number of pods per plant or number of seeds per pod at two locations .
\end{abstract}




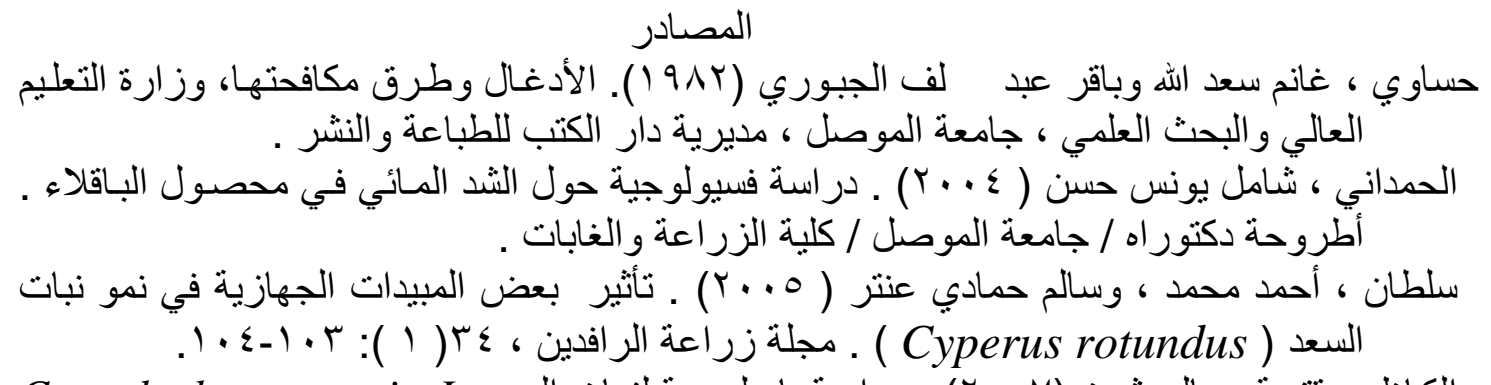

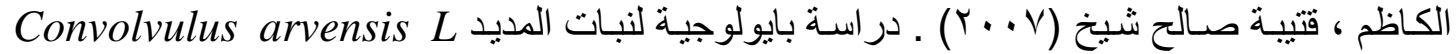

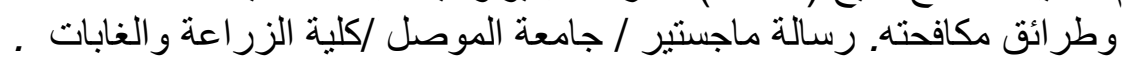

Abdel , C.G.(1997) . Physiological studies on growth flowering . Fruit setting and yield of Faba bean ( Vicia faba L.) ph .D.Thesis, Mosul univ - Iraq.

Loss , S.P.K;H.M.Siddique (1996) . Adaptation of faba bean to Mediterranean environments of western Australia, proceeding of the $8^{\text {th }}$. Australian Agronomy conterence, Toowoombo p 1-5.

Smith , F.R.( 2005)Cooperation extension co Acknowledgment for contributions to the weeds section .UC/PM pest management Cuidelines celery UC ANR publication 3439 Weeds The Regents of the univ of California Allfights reserved .

Summer field , R .J.and E.H.Roberts (1985) . Grain legume crops . Collins print .p 199-266.

Swisher, B.A. \& P.J. Shea (1985). Control of field bindweed with glyphosate plus additives. Proceedings North Central Weed Control Conference, p. 88.

Youtie, B.A. (1994). The Nature Conservancy Weed Report for the northeast Oregon Middle Fork John Day on Convolvulus arvensis L., field bindweed. TNC Wildland Weeds Management \& Research Weed Report. 